\title{
Pancreatic Cancer by AJCC v6 and v7 Stage
}

National Cancer Institute

\section{Source}

National Cancer Institute. Pancreatic Cancer by A/CC v6 and v7 Stage. NCI Thesaurus.

Code C134902.

A term that refers to the staging of exocrine and endocrine pancreatic cancer including well-differentiated neuroendocrine tumors according to the American Joint Committee on Cancer, 6th and 7th editions. 